\title{
Arborescences
}

Revue d'études françaises

\section{Le " bon usage du français au Québec " selon le Multidictionnaire de la langue française : le cas de la prononciation}

\section{Marie-Hélène Côté et Wim Remysen}

Numéro 7, décembre 2017

La norme orale en français laurentien

URI : https://id.erudit.org/iderudit/1050967ar

DOI : https://doi.org/10.7202/1050967ar

Aller au sommaire du numéro

Éditeur(s)

Département d'études françaises, Université de Toronto

ISSN

1925-5357 (numérique)

Découvrir la revue

Citer cet article

Côté, M.-H. \& Remysen, W. (2017). Le « bon usage du français au Québec » selon le Multidictionnaire de la langue française : le cas de la prononciation.

Arborescences, (7), 33-48. https://doi.org/10.7202/1050967ar
Résumé de l'article

Depuis son apparition sur le marché des dictionnaires au Québec, le Multidictionnaire de la langue française a suscité un intérêt particulier auprès du grand public, qui l'a rapidement adopté comme ouvrage de référence. Ce dictionnaire n'est toutefois pas à l'abri de tout reproche et, malgré ses prétentions, il ne rend que partiellement compte de l'usage tel qu'il a cours au Québec. Dans cet article, nous porterons un regard critique sur le traitement que le Multi réserve à la prononciation québécoise qui, contrairement au lexique, n’a pas été analysée par les linguistes qui se sont intéressés à l'ouvrage. Comme c'est le cas pour le lexique, la prise en compte très partielle de l'usage québécois en matière de prononciation soulève la question de la représentativité de ce dictionnaire et, partant, de son rôle comme outil de référence.
Tous droits réservés @ Département d'études françaises, Université de Toronto, 2018
Cedocument est protégé par la loi sur le droit d'auteur. L'utilisation des services d’Érudit (y compris la reproduction) est assujettie à sa politique d'utilisation que vous pouvez consulter en ligne. 


\section{SOMMAIRE}

1 Marie-Hélène Côté, Université de Lausanne

Anne-José Villeneuve, Université de l'Alberta Introduction

17 Davy Bigot, Université Concordia

Regard rétrospectif sur la norme du français québécois oral

33 Marie-Hélène Côté, Université de Lausanne

Wim Remysen, Université de Sherbrooke

Le "bon usage du français au Québec» selon le Multidictionnaire de la langue française: le cas de la prononciation

49 Anne-José Villeneuve, Université de l'Alberta

Normes objectives et variation socio-stylistique: le français québécois parlé en contexte d'entrevues télévisées

67 Monelle Guertin, Université du Québec à Montréal

Variation sociophonétique dialectale et stylistique: quelle est la langue cible en français langue seconde à Montréal?

90 Suzie Beaulieu, Université Laval

Leif French, Sam Houston State University

Samuel Gagnon, Université Laval

I'veulent-tu parler comme nous-autres? Opinions d'apprenants sur la forme interrogative totale en français laurentien 


\title{
Le «bon usage du français au Québec» selon le Multidictionnaire de la langue française: le cas de la prononciation
}

\author{
Marie-Hélène Côté, Université de Lausanne \\ Wim Remysen, Université de Sherbrooke
}

\section{Résumé}

Depuis son apparition sur le marché des dictionnaires au Québec, le Multidictionnaire de la langue française a suscité un intérêt particulier auprès du grand public, qui l'a rapidement adopté comme ouvrage de référence. Ce dictionnaire n'est toutefois pas à l'abri de tout reproche et, malgré ses prétentions, il ne rend que partiellement compte de l'usage tel qu'il a cours au Québec. Dans cet article, nous porterons un regard critique sur le traitement que le Multi réserve à la prononciation québécoise qui, contrairement au lexique, n’a pas été analysée par les linguistes qui se sont intéressés à l'ouvrage. Comme c’est le cas pour le lexique, la prise en compte très partielle de l'usage québécois en matière de prononciation soulève la question de la représentativité de ce dictionnaire et, partant, de son rôle comme outil de référence.

\section{Introduction}

Il est aujourd'hui évident que les Québécois valorisent une norme de prononciation qui diffère, sur bien des points, de celle qui a cours dans les autres pays francophones, notamment dans la francophonie européenne. Plusieurs études récentes, menées dans le domaine de la dialectologie perceptuelle et celui des représentations linguistiques, montrent en effet que l'accent le plus positivement évalué par les Québécois, ou encore jugé le plus apte pour l'enseignement du français au Québec, est celui que l'on peut entendre dans la bouche des locuteurs québécois scolarisés ${ }^{1}$. Si l'appartenance de certaines variantes de prononciation au registre soigné ou standard fait encore l'objet de débats (voir Bigot et Papen 2013), il existe clairement un modèle de prononciation propre au Québec.

Apparu dans les années suivant la Révolution tranquille et diffusé entre autres grâce à la radio et à la télévision publiques, ce modèle est le résultat d'un rapprochement relatif avec le modèle de prononciation européen que les correcteurs de la langue au Québec ont promu, dès le début du Xx ${ }^{\mathrm{e}}$ siècle, à travers la publication d'une série de manuels consacrés à la prononciation (Gendron 2014). En même temps, si la promotion de ce modèle a pu favoriser le recul de certains traits de prononciation traditionnellement attestés en français québécois, la norme de prononciation actuellement valorisée par les Québécois n'est pas en tout point conforme à la norme orale européenne, mais a plutôt fini par

1. À ce propos, voir entre autres l'étude de Moreau et al. (2007) qui observent que «les auditeurs québécois accordent un avantage net à leurs variétés propres, de façon consensuelle lorsque sont concernés les enregistrements de leurs socioculturellement dominants» (p. 42). D'autres études, adoptant des méthodologies différentes, arrivent à des constats semblables; voir par exemple Evans (2002) et Bouchard et al. (2004). 
intégrer certains traits qui leur sont spécifiques. C'est d'ailleurs ce modèle résolument québécois qui sera recommandé, en 1977, par l'Association québécoise des professeurs de français.

L'explicitation de cette norme phonétique n'est toutefois pas acquise et les Québécois ne disposent pas, à l'heure actuelle, d'un manuel de référence en matière de prononciation qui pourrait servir, entre autres, dans le milieu de l'enseignement ou dans le domaine des communications ${ }^{2}$. Ils doivent donc se tourner vers les dictionnaires décrivant l'usage québécois, dont certains rendent compte, quoique à des degrés variables, de l'usage en matière de prononciation. L'objectif de cet article est d'analyser ce qu'il en est dans l'ouvrage qui sert le plus souvent de référence pour la norme au Québec, le Multidictionnaire. Malgré sa popularité dans les milieux scolaires et langagiers, ce dictionnaire comporte plusieurs lacunes qui lui ont valu de sérieuses critiques ${ }^{3}$ et il véhicule, de l'avis de plusieurs linguistes, une image inexacte de l'usage québécois. Pour ce qui est de la prononciation, il s'agit d'un aspect qui est complètement passé sous silence dans les comptes rendus et critiques de l'ouvrage, d'où notre intérêt ${ }^{4}$. Après avoir présenté brièvement le Multi (section 2), nous analyserons la place qu'il accorde globalement à la prononciation (section 3), les traits phonétiques qui reçoivent une attention particulière dans les notes sur la prononciation (section 4) ainsi que la prise en compte de l'usage québécois (section 5).

\section{Le Multidictionnaire, un dictionnaire pas comme les autres}

Depuis son apparition en 1988, le Multidictionnaire de la langue française s'est taillé une place de choix sur le marché des dictionnaires au Québec et il s'est rapidement imposé comme référence en matière de norme prescriptive dans le milieu de l'enseignement, comme en fait foi l'approbation officielle de l'ouvrage pour l'enseignement secondaire dès 1989. Si les cinq rééditions de l'ouvrage, publiées successivement entre 1988 et 2015, confirment la popularité du Multidictionnaire, elles témoignent aussi d'une importante évolution dans sa vocation et sa conception même. Conçu d'abord comme un dictionnaire spécialisé consacré aux "difficultés linguistiques des usagers du français au Québec» (De Villers 1988 : xv), l'ouvrage s'est progressivement donné pour ambition de " [décrire] le français de tous les francophones", particulièrement à partir de la troisième édition, publiée en 1997. L'ouvrage prend ainsi de plus en plus les allures d'un dictionnaire général usuel, dont l'objectif est de traiter globalement le lexique de la langue française:

La nomenclature du Multidictionnaire est constituée de la plupart des mots courants du français contemporain, à l'exception des termes très techniques ou scientifiques. [...] En vue d'éviter au lecteur la consultation de nombreux ouvrages, il a semblé plus pratique de traiter l'ensemble des mots usuels et de mettre en évidence tous les types de renseignements linguistiques qui leur sont propres, indépendamment de leur nature. (De Villers 2015: xiii)

2. Deux initiatives récentes méritent néanmoins d'être soulignées: le manuel Les prononciations du français québécois (Ostiguy et Tousignant 2008) et la base Phono: les principales caractéristiques du français parlé au Québec (Paradis, Dolbec et Arnaud 2008). Les deux proposent un inventaire des particularités phonétiques et phonologiques du français en usage au Québec et accordent une importance particulière aux variations sociolinguistiques qui touchent la prononciation au Québec, d'où leur utilité pour les enseignants et les communicateurs.

3. Outre l'orientation normative ambivalente, sur laquelle nous reviendrons plus loin, les critiques ont essentiellement porté sur les définitions imprécises et lacunaires de l'ouvrage (cf. Séguin 2013, qui a analysé la pratique définitoire d'un champ lexical précis, celui des noms de plantes alimentaires).

4. À propos du traitement de la prononciation dans d'autres dictionnaires conçus au Québec, voir Thibault et Thibault (1993) et Thibault (2013). 
D'une édition à l'autre, cette transformation se traduit non seulement par un accroissement de la nomenclature, mais aussi par un enrichissement progressif de la description lexicographique (définitions systématiques et plus élaborées, intégration de collocations, de synonymes et d'antonymes, exemplification et illustrations littéraires, etc.; voir Cormier 2008; Séguin 2013). En même temps, le dictionnaire n'a jamais abandonné sa vocation première d'être un guide normatif, ce que montrent bien les nombreux tableaux thématiques dont il est agrémenté et qui fournissent à l'utilisateur des informations à propos de certaines difficultés grammaticales, orthographiques et typographiques. Sa dimension corrective se traduit aussi par le traitement réservé aux mots qui composent la nomenclature et dont l'auteure cherche souvent à orienter l'usage, notamment par le recours à l'astérisque pour signaler les «formes fautives ${ }^{5} »$.

Cette orientation particulière du Multidictionnaire, qui combine description et correction, explique sans doute le succès que l'ouvrage connaît auprès des utilisateurs, qui préfèrent souvent les prises de position tranchées aux explications nuancées. Aux yeux de Jean-Claude Corbeil, directeur linguistique du projet, le pragmatisme du Multi, nom sous lequel il est maintenant largement connu du public, sert mieux l'utilisateur qui a besoin de trouver rapidement une réponse claire et univoque à ses questions :

[...] le MULTI se veut d'une consultation rapide, pour que le lecteur y trouve réponse à ses questions d'un coup d'œil, immédiatement, dans le feu de l'action pour ainsi dire. D'où une mise en page qui va directement à l'essentiel, qui fait le sacrifice des longues explications, auxquelles il substitue des étiquettes connues et compréhensibles, explicitées si nécessaire par une courte note et souvent symbolisées par l'ajout de différents icônes. (Corbeil 2004: 27)

L'orientation normative du Multi, au cœur du projet lexicographique, est pourtant problématique (Jousselin 1989; Bergeron 1998; Poirier et Canac-Marquis 2003; Poirier 2004). C'est que tout en prétendant de "proposer la description la plus fidèle du bon usage du français au Québec» (De Villers 2015: xiii), l'auteure condamne bien des emplois qui sont tout à fait standard dans l'usage québécois. L'auteure ne voit d'ailleurs aucune contradiction entre la mission qu'elle se donne de décrire l'usage qui a cours au Québec et, dans le même temps, celle de "[décrire] le français standard, tel qu’il est explicité par les grammaires et les grands dictionnaires, noyau dur de la langue française commune à tous les francophones" (Corbeil 2015: xii). Les critiques formulées à l'endroit du Multi ont surtout porté sur la composante lexicale de l'ouvrage. Dans cet article, il sera question plutôt de la prononciation québécoise, qui fait partie des «difficultés» de la langue abordées dans l'ouvrage.

\section{Le traitement de la prononciation dans le Multidictionnaire: aperçu général}

La prononciation n'occupe pas une place de choix dans le Multi, où les mots ne sont pas systématiquement accompagnés d'une transcription phonétique. Les seules informations de nature phonétique figurent dans des «notes sur la prononciation», introduites par le symbole " $\boldsymbol{\nabla}$ ". Ces notes accompagnent des mots dont la prononciation est, pour diverses raisons sur lesquelles nous reviendrons

5. Il arrive aussi que l'auteure proscrive indirectement certains emplois (p. ex. lorsqu'elle précise, dans l'article buanderie, qu'on se sert du mot blanchisserie "[pour] désigner l'établissement commercial qui se charge de blanchir le linge», condamnant dans les faits l'usage que les Québécois font de buanderie). 
plus loin, jugée problématique: "Les mots difficiles à prononcer ou les mots d'origine étrangère sont accompagnés d'une indication en toutes lettres ainsi que de leur transcription entre crochets selon les critères de l'Association phonétique internationale (API)» (De Villers 2015: xviii). L'introduction reste muette sur les critères de sélection qui ont guidé l'auteure dans le choix des cas jugés «difficiles» ainsi que sur les principes qui orientent leur traitement normatif. On doit donc présumer que le même principe s'applique pour la délimitation de la norme phonétique que celui qui prévaut dans le cas de la norme lexicale: «le Multidictionnaire se donne pour mission de proposer la description la plus fidèle du bon usage du français au Québec» (De Villers, 2015: xiii).

À partir de la version électronique de la sixième édition du Multidictionnaire ${ }^{6}$, nous avons fait l'inventaire de l'ensemble des articles qui contiennent une note de prononciation. Ces articles sont au nombre de 2327, ce qui représente environ $5 \%$ de l'ensemble de la nomenclature. On note d'entrée de jeu que l'auteure de l'ouvrage recourt à cinq stratégies différentes pour rendre la prononciation d'un mot:

1. accompagner le mot d'une transcription en alphabet phonétique international, comme escient "[a]ttention à la prononciation, [esjã ]», quolibet «se prononce [kolibe]» (c'est le cas dans 2170 articles);

2. expliquer certains phénomènes de prononciation par le recours à un langage métalinguistique (qui peut être plus ou moins technique), comme dans le cas de dossier, "[l]e $o$ est fermé» et mambo «[l]e deuxième $m$ est sonore» (1 290 articles);

3. miser sur la correspondance graphème/phonème, comme dans le cas de minutieux, "[1]e $t$ se prononce s» ou de céleri/cèleri, "[1] e é se prononce è, parfois é» (948 articles);

4. faire rimer (ou comparer) les mots qui posent problème avec des mots dont la prononciation est jugée mieux connue de l'utilisateur, comme pour génome "rime avec dome», bagel/baguel "[l]e $g$ se prononce comme dans baguette» (539 articles);

5. pour les emprunts, associer la prononciation à la langue source ou à la langue cible (ici le français), comme pour mojito «se prononce à l'espagnole", scrabble "se prononce à l'anglaise ou à la française, [skrabəl, skrabl]» (51 articles).

En règle générale, l'auteure recourt à plusieurs stratégies à la fois, comme le montre l'exemple tobbogan "[l] es $o$ sont ouverts et le $n$ est muet, [tobogã] ; le mot rime avec gant", qui inclut le métalangage (2), la rime (4) et la transcription (1), cette dernière étant pratiquement automatique à la fin de chaque note. Il faut aussi noter que les mêmes phénomènes ne sont pas traités de façon systématique; par exemple la prononciation de $<\mathrm{um}>^{7}$ en [om] est signalée d'au moins trois façons différentes, en plus du recours à la transcription phonétique: "[l] e $u$ se prononce comme un $o$ ouvert» (décorum) (2), «[1]a lettre $u$ se prononce $o$ » (album) (3), "rime avec gomme» (caécum) (4).

Le recours à la rime est une astuce intéressante qui a une valeur pédagogique certaine, les transcriptions phonétiques n'étant pas facilement interprétables pour des non-initiés (et a fortiori pour des élèves en situation d'apprentissage). Les rimes peuvent toutefois être problématiques et manquer leur but. C'est le cas lorsqu'elles reposent sur la prononciation européenne plutôt que québécoise, comme pour dilemme "[l]e nom rime avec problème», alors qu'au Québec il rime plutôt avec crème. D’autres

6. Cette version électronique, disponible à l'adresse www.multidictionnaire.com, date de mai 2015. Elle reprend intégralement le contenu de la sixième édition de la version papier, publiée en avril de la même année.

7. Les chevrons $<\ldots>$ indiquent des séquences orthographiques. 
problèmes surgissent lorsque le mot servant de comparaison est rare ou potentiellement peu connu de l'utilisateur: «le mot [fez] rime avec laize», «le mot [stop] rime avec isotope».

Par ailleurs, l'inventaire des symboles de l'API retenu pour les transcriptions est celui qu'on trouve dans des dictionnaires de français généraux, comme le Petit Robert ${ }^{8}$. Or cet inventaire ne correspond pas au système phonémique du français québécois, donc il ne peut pas, dans bien des cas, rendre compte des spécificités de l'usage du Québec, malgré les prétentions du Multi. Cet aspect sera développé dans la section 5 .

La référence à la langue source ou cible pour la prononciation des emprunts est encore plus problématique car on ne sait pas exactement ce qu'est une prononciation «à la française», "à l'anglaise» ou «à l'espagnole». L'ajout d'une transcription phonétique clarifie la prononciation du mot concerné, mais elle n'est pas toujours présente, comme pour mojito, prononcé «à l'espagnole», et on ne peut pas toujours dire qu'elle soit conforme à la langue donnée. Par exemple, muffin «se prononce à l'anglaise, [mœfin]». On peut penser que cette prononciation s'oppose à une version à la française qui serait [myf̃̃], mais on peut difficilement considérer que les voyelles [œ] et [i] relèvent d'une prononciation à l'anglaise. La transcription suggérée [mœfin] correspond à la prononciation européenne, par opposition à [mofyn] ou [mofœn] au Québec.

\section{Les «difficultés de prononciation»: typologie}

Le Mutidictionnaire a choisi de ne noter la prononciation que pour un sous-ensemble des mots inventoriés: "mots difficiles à prononcer» ou «mots empruntés à d'autres langues» (p. xiv). Si la difficulté que peut présenter la prononciation des emprunts se conçoit aisément, les «mots difficiles à prononcer» ne sont nulle part définis. Afin de circonscrire cette notion de difficulté, nous avons relevé pour chacune des 2327 notes de prononciation le ou les trait(s) de prononciation ciblé(s), en distinguant les traits explicites - qui font l'objet d'un commentaire spécifique - et les traits implicites - que l'on peut seulement dégager des transcriptions phonétiques. Par exemple, le mot yucca est assorti de la note "[l] e $u$ se prononce $o u$, [juka] ». Le trait explicite est ici la prononciation de $<\mathrm{u}\rangle$, mais la transcription recommande aussi implicitement le [a] antérieur final plutôt que le [a] postérieur caractéristique de la prononciation québécoise?

On dénombre 2409 traits de prononciation explicitement mentionnés ${ }^{10}$. Dans plusieurs cas, le même trait s'applique à plus d'un mot de la même famille; par exemple, la prononciation de $<\mathrm{t}>$ en

8. À noter que le Multidictionnaire n'inclut pas dans son inventaire le symbole [x] (utilisé dans le Petit Robert pour rendre compte de la prononciation de certains mots empruntés à l'espagnol, comme mojito). Autre particularité, le Multidictionnaire utilise systématiquement [r] pour la prononciation du /R/, le symbole [r] étant parfois utilisé par défaut pour la transcription de la consonne rhotique, même s'il représente selon l'API une variante apicale.

9. Les traits implicites exprimés par les transcriptions sont potentiellement nombreux et d'intérêt variable et il n'est pas envisageable de les noter tous ou d'identifier les seuls traits pertinents. Pour yucca, le [a] final est intéressant, mais la correspondance entre $<\mathrm{cc}>$ et $[\mathrm{k}]$ l'est sans doute moins, puisque $<\mathrm{c}>$ ne se prononce jamais autrement que $[\mathrm{k}]$ devant $<\mathrm{a}>$.

10. Plusieurs entrées incluent plus d'un trait explicite, d'autres (103) n'en contiennent aucun. C'est le cas de «ce mot se prononce» ou "attention à la prononciation», suivi d'une transcription phonétique, où aucun trait n’est spécifiquement ciblé. Les emprunts sont aussi régulièrement accompagnés de la mention «ce mot se prononce à l’anglaise/à la française/à l'espagnole»; il est cependant difficile d'identifier clairement un ou plusieurs traits de prononciation, cela d'autant plus que le caractère «anglais », «français» ou «espagnol» des prononciations peut être douteux, comme on l'a vu dans la section 3 . 
[s] est notée pour l'adjectif impatient, mais également pour les mots qui en sont dérivés (impatience, impatiemment). En ne comptant qu'une seule occurrence dans le cas d'un trait répété à l'identique dans plusieurs mots reliés (à condition que ces mots soient adjacents dans le dictionnaire), le nombre de traits mentionnés passe à 1999, qui se répartissent comme suit (voir tableau 1). Les exemples du tableau reprennent à l'identique les commentaires du Multi.

Tableau 1: Traits de prononciations explicites dans les notes de prononciation du Multi

\begin{tabular}{|c|c|c|c|c|}
\hline \multicolumn{2}{|c|}{ Catégories principales } & \multicolumn{2}{|l|}{ Sous-catégories } & Exemples \\
\hline \multirow{4}{*}{$\begin{array}{l}\text { Consonnes } \\
\text { muettes ou } \\
\text { prononcées }\end{array}$} & \multirow[t]{4}{*}{$\begin{array}{l}757 \\
(38 \%)\end{array}$} & C orales finales & 567 & $\begin{array}{l}\text { trafic: Le } c \text { se prononce [trafik] } \\
\text { rot: Le } t \text { ne se prononce pas [ro] }\end{array}$ \\
\hline & & C nasales & 119 & $\begin{array}{l}\text { amen: Le } n \text { se prononce [amen] } \\
\text { pampa: La première syllabe se prononce pan [pãpa] }\end{array}$ \\
\hline & & C orales non finales & 50 & $\begin{array}{l}\text { amygdale: On ne prononce pas le } g \text { [amidal] } \\
\text { dompter: Le } p \text { se prononce ou non }[\mathrm{d} \tilde{\mathrm{s}}(\mathrm{p}) \mathrm{te}]\end{array}$ \\
\hline & & Liaison & 21 & avant-hier: On fait la liaison [avãtjer] \\
\hline \multirow[t]{5}{*}{$\begin{array}{l}\text { Timbre des } \\
\text { voyelles }\end{array}$} & \multirow[t]{5}{*}{$\begin{array}{l}593 \\
(30 \%)\end{array}$} & V à double timbre & 276 & $\begin{array}{l}\text { coma: Le } o \text { est ouvert [koma] } \\
\text { effarant: Le } e \text { se prononce } e ́\left(\text { et non }{ }^{*} \dot{e}\right)\end{array}$ \\
\hline & & $<\mathrm{e}>$ & 124 & $\begin{array}{l}\text { veto: Le } e \text { se prononce } e ́[\text { veto] } \\
\text { registre: Attention à la première syllabe qui se prononce re } \\
\left(\text { et non }{ }^{*} \text { ré), [rəzistr] }\right.\end{array}$ \\
\hline & & Emprunts & 109 & $\begin{array}{l}\text { sir: Le } i \text { se prononce eu. Le nom rime avec sœur [sœr] } \\
\text { crawl: Les lettres aw se prononcent } o \text {, [krol] }\end{array}$ \\
\hline & & V nasales & 35 & $\begin{array}{l}\text { jungle: Les lettres un se prononcent un ou on [30̃egl, 30̃gl] } \\
\text { appendice: Les lettres en se prononcent in [apẽdis] }\end{array}$ \\
\hline & & Digrammes & 49 & $\begin{array}{l}\text { poil: Le } i \text { se prononce comme un } a \text { [pwal] } \\
\text { faisan: Les lettres } a i \text { se prononcent } e[\text { fəzã }] \text {, comme dans fenêtre }\end{array}$ \\
\hline $\begin{array}{l}\text { Timbre des } \\
\text { consonnes }\end{array}$ & $\begin{array}{l}363 \\
(18 \%)\end{array}$ & \multicolumn{3}{|c|}{$\begin{array}{l}\text { ratio: Le } t \text { se prononce } s \text { [rasjo] } \\
\text { quantum: La première syllabe se prononce kan ou kouan [kãtom, kwãtom] } \\
\text { désuet: Le } s \text { se prononce } z \text { ou } s \text { [dezप } \varepsilon \text {, desप } \varepsilon]\end{array}$} \\
\hline $\begin{array}{l}\text { Schwa } \\
\text { muet ou } \\
\text { prononcé }\end{array}$ & $\begin{array}{l}159 \\
(8 \%)\end{array}$ & \multicolumn{3}{|c|}{$\begin{array}{l}\text { fausseté: Le } e \text { central ne se prononce pas [foste }] \\
\text { chenapan: Le } e \text { se prononce ou non }\left[\int(\partial) \text { napã }\right]\end{array}$} \\
\hline $\begin{array}{l}\text { Séquences } \\
\text { particulières }\end{array}$ & $\begin{array}{l}88 \\
(4 \%)\end{array}$ & \multicolumn{3}{|c|}{$\begin{array}{l}\text { joker: Le } r \text { est sonore [3oker, dzoker] } \\
\text { atrium: Les lettres um se prononcent omme [atrijom] }\end{array}$} \\
\hline Autres & $39(2 \%)$ & \multicolumn{3}{|c|}{ 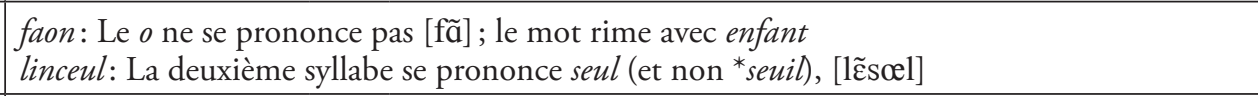 } \\
\hline TOTAL & 1999 & & & \\
\hline
\end{tabular}

Les deux principales catégories de traits, qui en regroupent les deux tiers (68\%), concernent le statut muet ou prononcé des consonnes et le timbre des voyelles. Pour la prononciation des consonnes, il s'agit essentiellement des consonnes finales, comme dans talus et tennis, où le $<s>$ est, respectivement, muet et prononcé. Les consonnes internes de mot, comme dans damné $(<\mathrm{m}>$ muet) et arctique ( $<\mathrm{c}>$ prononcé) sont beaucoup plus rarement mentionnées. Les consonnes nasales, internes ou finales, forment un cas à part, la réalisation de la consonne déterminant également le timbre de la voyelle précédente (p. ex. < an > est réalisé [an] ou [ã]). On trouve enfin quelques commentaires portant sur la liaison, notamment avec les chiffres (p. ex. huit) et dans certains mots composés (p. ex. pied-à-terre). 
Le timbre des voyelles concerne un ensemble varié de phénomènes, dont on peut cependant faire ressortir les sous-catégories suivantes:

- La prononciation des voyelles dites à «double timbre» $[\mathrm{e} \sim \varepsilon],[\varnothing \sim \propto],[\mathrm{o} \sim 0]$ et $[\mathrm{a} \sim \mathrm{a}]$, comme dans aiguille, peuplier, encyclopédie et caillot. On précise surtout le caractère ouvert ou fermé de $<$ o $>$ et $<$ e $>$ (plus rarement $<$ au $>$ et $<$ ai $>$ ) dans respectivement 182 et 89 commentaires. Dans la grande majorité de ces cas, il s'agit de voyelles en syllabe non finale.

- Le timbre des voyelles nasales, notamment le cas de $<$ en $>$ prononcé [ $\widetilde{\varepsilon}]$, comme dans agenda.

- La réalisation du < e > non accentué en [ə], [e] ou [a], comme dans ressac [ə], feta [e] ou différemment $[\mathrm{a}]$.

- Le timbre des voyelles dans les emprunts (outre les cas de $<$ e > prononcé [e]). Cela inclut toute une variété d'adaptations vocaliques de mots empruntés, surtout de l'anglais, par exemple $<\mathrm{a}>$ réalisé $[\varepsilon]$ (p. ex. ale) ou [o] (p. ex. hall), <u> prononcé [u] (p. ex. burka/burqa) ou [œ] (p. ex. chutney) et $<\mathrm{ie}>$ prononcé $[\mathrm{i}]$ (p. ex. lied).

- La prononciation de différents digrammes (p. ex. <oi > prononcé [wa] dans coi; < ai > et $<$ on $>$ prononcés [ə] dans satisfaisant, monsieur; < ay > prononcé [عj] dans paye, etc.).

La réalisation variable de différentes consonnes (ou digrammes) rend compte de $18 \%$ des commentaires explicites. Les emprunts sont ici aussi largement mis à contribution. Cela inclut la prononciation de $<\mathrm{ch}>$ en [k] ou [t $\mathrm{f}$ ] plutôt que [ $\mathrm{S}]$ (84 cas, p. ex. orchidée, gaspacho), $<\mathrm{t}>$ en [s] plutôt que [t] (57 cas, p. ex. ratio), < qu > en [k], [kw] ou [ku] (55 cas, p. ex. équestre, équateur, équidistant) et $<1 \mathrm{ll}>$ en [j] ou [1] (39 cas, p. ex. millet, allô), ainsi que la réalisation $\mathrm{de}\langle\mathrm{g}\rangle,\langle$ gu $\rangle,\langle$ gn $\rangle,\langle\mathrm{c}(\mathrm{c})\rangle,\langle\mathrm{s}(\mathrm{c})\rangle,\langle\mathrm{w}\rangle$, $<\mathrm{x}>$ et $<\mathrm{z}(\mathrm{z})>$. On note également neuf mentions reliées au voisement des consonnes, comme le $<\mathrm{b}$ $>$ de abcès prononcé $[\mathrm{p}]$.

L'omission ou le maintien du e muet fait l'objet de 157 commentaires, soit $8 \%$ du total. Dans la plupart des cas, c'est l'omission d'un $<$ e $>$ en syllabe médiane qui est visée, comme dans paletot. Le reste des notes de prononciation porte sur deux séquences particulières $-<\mathrm{um}>$ prononcé [om] $(51$ cas, p. ex. pensum) et <er > prononcé [Er] ou [œr] (et non [e]) (37 cas, p. ex. polder) - et sur un éventail de traits marginaux (39 cas) allant de l'absence de gémination dans méchamment à des mises en garde contre des prononciations telles que [agisabl] pour haïssable ou [asteriks] pour astérisque.

Ces traits ne sont toutefois pas notés systématiquement. Pour chaque mot pour lequel on note un trait de prononciation, il est aisé d'en trouver un comparable qui ne fait l'objet d'aucun commentaire. Par exemple, si l'absence du schwa en syllabe médiane est notée dans de nombreux cas (ex. fièrement, follement), elle ne l'est pas dans d'autres (ex. doucement, grassement). L'omission variable du schwa en syllabe initiale touche en français plusieurs centaines de mots (Côté 2009), mais le Multi ne la mentionne que dans douze d'entre eux: pourquoi chenil, genou ou pelouse, mais pas cheville, gelée ou semaine? De même, le timbre ouvert $\mathrm{du}<\mathrm{o}>$ est noté dans astrologue, géologue et sept autres mots en -ologue, mais pneumologue, ethnologue et autres cardiologue ne portent aucune mention. Si la séquence $<\mathrm{um}>$ se prononce [om] dans aquarium et uranium, rien n'indique que ce soit aussi le cas pour harmonium et hélium. Et ainsi pour chacun des traits de prononciation relevés dans le tableau 1.

Un cas particulier d'incohérence est celui des groupes consonne + liquide en fin de mot. Ces groupes, très fréquents, sont régulièrement simplifiés par la chute de la liquide dans toutes les variétés familières de français (p. ex. table [tab], pitre [pit]; voir Milne 2014 et Boughton 2015). Le Multidictionnaire met le lecteur en garde contre ce phénomène au mot sucre: «[l]e $r$ se prononce, [sykr], (et non ${ }^{*}$ suc)». Mais il reste muet pour les centaines d'autres mots du même type. 
Les emprunts, auxquels le Multi porte une attention particulière, ne sont pas traités de façon plus systématique: volleyball mais pas basketball, rugby mais pas soccer ou hockey, hamburger mais pas hotdog, cocker mais pas husky, alléluia mais pas mea culpa. Suspense, living(-room), hacienda ou halloween sont également dépourvus de note de prononciation, tout comme toutes les formes considérées comme fautives, souvent des anglicismes, qui ne sont jamais commentées. Un coup d'œil rapide aux entrées du dictionnaire révèle de nombreux autres mots susceptibles de soulever des questions de prononciation laissées sous silence, par exemple hayon ([a], [a] ou [ع] ?), ignifuge ([gn] ou [n] ?) ou lupus (avec ou sans $[\mathrm{s}]$ ?).

Au-delà de l'incohérence dans l'application des traits, on voit souvent mal ce qui justifie les notes de prononciation pour des mots qui, à notre connaissance, n'ont jamais posé problème. Pourquoi noter que allô se prononce avec un seul [1] et méchamment avec un seul [m] ou que la prononciation du verbe abrier rime avec briller? Faut-il préciser que les mots avis et brebis se prononcent sans [s], que le $<$ o $>$ de glotte ou volt est ouvert ou que les mots pinceau et sceau riment respectivement avec sot et seau? La prononciation de $<\mathrm{t}>$ en $[\mathrm{s}$ ] dans conviction, impatient, initial et de nombreux autres représente-t-elle une difficulté de prononciation ${ }^{11}$ ?

On peut aussi s'interroger sur l'insistance mise sur les voyelles à double timbre, notamment [e $\sim \varepsilon]$ et [o o] qui, à elles seules, font l'objet de $14 \%$ des traits explicitement mentionnés. La distinction est certainement pertinente en syllabe finale, par exemple dans pathos, synchrone ou tome, où on peut noter une hésitation entre [o] et [o]. Mais dans la très large majorité des cas, c'est en syllabe non finale que le timbre de la voyelle est noté, dans une position où la distinction est souvent moins que claire et peut être considérée comme secondaire, voire absente. De façon générale, le Multi recommande [ 0 ] plutôt que $[\mathrm{o}]$ et [e] plutôt que $[\varepsilon]$, alors que l'usage québécois tend souvent vers l'autre voyelle. Mais le caractère plus ou moins ouvert des $<\mathrm{o}>$ de philosophie, togolais ou robotique et des $<\mathrm{e}>$ de effroi, cellophane ou greffé est-il susceptible de nuire à la reconnaissance lexicale ou de suggérer une maîtrise imparfaite du français? La prononciation des voyelles moyennes illustre particulièrement bien la frilosité du Multi face à la variation, sans compter les cas où le dictionnaire recommande des prononciations qui s'opposent clairement à l'usage québécois, comme dans coma, doper, vomi, soda, smoking et sofa, où on recommande [0], en opposition au [o] québécois.

Il faut convenir que la notion de «difficulté de prononciation» est plutôt opaque et que son traitement manque largement de cohérence. Comme on le verra plus en détail dans la section suivante, les "difficultés» incluent de nombreux particularismes québécois: dans une large mesure, les notes de prononciation concernent précisément les mots qui ont une prononciation différente au Québec et pour lesquels on recommande la prononciation «française».

\section{L'usage et le bon usage québécois en matière de prononciation: regard critique}

Pour chacune des entrées qui fait l'objet d'une note de prononciation, nous avons également comparé les recommandations du Multidictionnaire avec, d'une part, les usages qui nous semblent dominants au Québec et, d'autre part, les transcriptions du Petit Robert (désormais PR) numérique (édition 2016).

11. De telles remarques pourraient être utiles pour des apprenants de français langue seconde, mais il est clair que les notes de prononciation ne visent pas ce groupe de locuteurs, étant donné la nature des commentaires et la faible proportion des mots ciblés. 
Ce sont ces différents codages qui vont nourrir la suite de la discussion, en commençant par la place qui est faite dans les notes à la variation géographique qui caractérise la prononciation en français.

\subsection{La prise en compte de la variation géographique}

Malgré son objectif de décrire "le bon usage québécois», le Multi témoigne de peu de sensibilité à l'endroit des spécificités québécoises en matière de prononciation. Contrairement aux québécismes lexicaux, qui sont identifiés par la fleur de lys, les particularismes phonétiques ne sont en effet pas signalés comme tels. Seulement trois notes mentionnent explicitement l'existence d'une variante de prononciation différente au Québec (tableau 2). Et encore, la description de la variante québécoise est problématique dans les trois cas ${ }^{12}$.

Tableau 2: Prononciations présentées comme propres au Québec dans le Multi

\begin{tabular}{|l|l|}
\hline \multicolumn{1}{|c|}{ Article } & \multicolumn{1}{c|}{ Prononciation selon le Multi } \\
\hline juin & Le mot rime avec brin. Au Québec, le mot rime couramment avec brun. \\
\hline ketchup & $\begin{array}{l}\text { Le mot se prononce à l'anglaise, [ketfop, ket } \\
\text { comme dans quête, la seconde comme dans chope. }\end{array}$ \\
\hline $\begin{array}{l}\text { yogourt ou yoghourt } \\
\text { ou yaourt }\end{array}$ & $\begin{array}{l}\text { Au Québec, le nom se prononière syllabe se prononce } \\
\text { prononce surtout ya-ourt (avec ou sans } t \text { final), [jogur, jaurt, jaur]. }\end{array}$ \\
\hline
\end{tabular}

Cela ne signifie pas pour autant que le Multi passe sous silence tous les traits de prononciation québécois. Dans certains cas, les transcriptions phonétiques rendent bien compte de la prononciation soignée que l'on peut entendre au Québec, comme pour les mots aồt [u], bobsleigh [bobsle], condom [kõd ̃̃] et consensus [kõs Ẽsys, kõsãsys], respectivement transcrits [u(t)], [bobsleg], [kõdom]

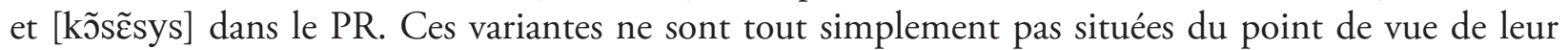
distribution géographique, ce qui n'est pas conforme à l'objectif que l'auteure se donne de "distinguer les usages qu'ont en partage tous les francophones de ceux qui sont propres au Québec et à la francophonie canadienne» (Introduction, p. xiii).

Dans les faits, les variantes québécoises et européennes cohabitent couramment dans le Multidictionnaire, non seulement d'un article à l'autre - certains commentaires contenant exclusivement des prononciations européennes, comme albatros [albatros] - mais aussi parfois à l'intérieur d'un seul et même article - pour soja/soya, on donne la prononciation avec [j] et [3], mais sans indiquer que la première est plus courante au Québec et la deuxième en France. Pour un dictionnaire qui se réclame de l'usage québécois, les transcriptions sont en réalité très similaires à celles qu'on peut trouver dans les dictionnaires français usuels les plus courants, comme le révèle la comparaison avec le PR. Des 2123 mots ${ }^{13}$ qui sont accompagnés d'une transcription phonétique dans les deux ouvrages, 1842 mots, soit

12. Pour juin, le commentaire fait d'abord rimer le mot avec brin et la légitimité de la variante québécoise n'est pas claire.

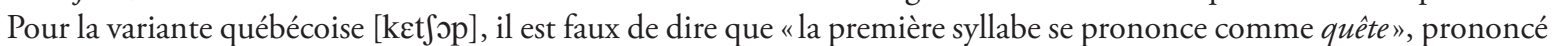
[k3t] et non [ket] (voir section 5.2 pour l'usage du symbole [3]). Quant à yoghourt, il est prononcé [jogur] plutôt que [jogur].

13. De tous les mots accompagnés d'une transcription phonétique dans le Multi (2 170), certains sont absents du Petit Robert - c'est le cas de quelques gentilés (bruxellois et togolais par exemple) et de quelques québécismes ou statalismes (comme zucchini et REER) - alors que d'autres n'y sont accompagnés d'aucune transcription phonétique -, c'est le cas de quelques préfixes (ichty(o)-, oxy-) ou encore de quelques adverbes qui ne font pas l'objet d'un article autonome, mais qui sont simplement mentionnés dans l'article consacré à l'adjectif dont ils dérivent, comme insatiablement et 
presque 9 cas sur 10, sont en effet transcrits de façon identique. Qui plus est, dans la grande majorité des cas où la prononciation québécoise s'écarte de celle qui est indiquée dans le PR, le Multidictionnaire ne prend pas la peine de refléter ces différences.

\subsection{Les différences systématiques entre les prononciations québécoise et européenne}

Nous avons mentionné dans la section 3 que le Multi ne tenait pas compte, dans son inventaire de symboles phonétiques, des spécificités du système phonémique québécois. D’une part, la distinction entre les voyelles de faites et de fête est totalement ignorée, même si elle est tout à fait stable dans l'usage québécois et intégrée à la norme de prononciation au Québec (Cox 1998). Seul le signe [ع] est donc utilisé, ce qui contraste avec le dictionnaire Usito (Cajolet-Laganière et Martel 2013) et le Dictionnaire historique du français québécois (Poirier 1998), qui utilisent respectivement [ع:] et [3] pour la voyelle de fête; c'est le symbole [3] que nous retiendrons ici ${ }^{14}$.

On ne sait pas si l'auteure du Multidictionnaire favorise implicitement un alignement sur la norme parisienne par la neutralisation de cette distinction historique ou si elle en ignore l'existence ou le statut dans le système de prononciation. Toujours est-il qu'une grande quantité de mots systématiquement prononcés avec [3] au Québec sont transcrits [ع] ou [e] dans le Multi: [e] dans blêmir, bêtise, évêché, grêler, guêpier, sherry, $[\varepsilon]$ dans grêlon, faciès, confesse ${ }_{n o m}$, enneigé, in-seize, merguez, palmarès, pataquès, xérès, estrogène, schizophrène, faîte, obscène (les trois derniers mots présentant plutôt une variation entre [ع] et [3] au Québec).

Un problème plus périphérique mais tout de même réel se pose pour les voyelles fermées. Les Québécois distinguent deux séries de voyelles fermées, tendues et relâchées (ou longues et courtes), qui établissent une opposition entre cool et coule, frise et quiz ou les deux voyelles de beatnik [bitnık] (Côté 2012). Le Multidictionnaire ne peut exprimer cette distinction et transcrit jeep, bit et beatnik avec la même voyelle $[\mathrm{i}]^{15}$.

Au-delà de l'inventaire phonémique, les usages québécois présentent des différences systématiques par rapport aux prononciations rapportées dans les dictionnaires généraux comme le PR. On peut donc se demander dans quelle mesure le Multi reflète ces spécificités. Nous avons retenu pour analyse la distribution des voyelles ouvertes en syllabe finale, celle des voyelles moyennes en syllabe non finale et la réalisation des voyelles fermées devant voyelle. Nous nous pencherons également sur certaines tendances bien établies dans l'adaptation des emprunts de l'anglais.

maussadement. Nous avons aussi mis de côté les quelques cas concernant la liaison car le phénomène n'est pas traité de la même façon dans les deux ouvrages.

14. Voir Côté (2012) pour une justification de ce symbole. L'utilisation de [3] plutôt que [ع:] souligne le timbre nettement distinct au Québec des voyelles de fête et faites, indépendamment de la différence de durée. La situation est comparable pour les voyelles de patte [a] et pâte $[\mathrm{a}]$ et elle permet de faire reposer l'ensemble du système vocalique sur des oppositions de timbre.

15. L'affrication de $[\mathrm{t} \mathrm{d}]$ devant $[\mathrm{i}$ y $\mathrm{j}$ $]$, qui est également intégrée dans la norme de prononciation du français au Québec, n'est pas notée non plus dans les transcriptions. Le dictionnaire Usito a fait le même choix et cela peut se justifier par la systématicité du processus en français québécois. Cela soulève tout de même le problème des exceptions lexicales, comme building ou meeting, où l'affrication ne s'applique le plus souvent pas, ce qu'il n'est pas possible de rendre dans un système de transcription qui ignore le phénomène. 
Les dictionnaires français et l'usage québécois s'accordent sur l'existence d'une opposition entre [a] et [a]. En syllabe finale, la distribution des deux voyelles en français québécois ne correspond cependant pas à l'usage signalé dans le PR. Le français québécois maintient une opposition entre [wa] et [wa] pour le digramme < oi >, tant en syllabe finale ouverte (ex. moi vs mois, bois verbe $_{\text {ve }}$ vois${ }_{n o m}$ ) que fermée (ex. boite ${ }_{\text {verbe }}$ vs boitte ${ }_{\text {nom }}$, poil vs poêle). Dans le PR, cette opposition est neutralisée en faveur de [wa]. La séquence < oi > exceptée, le PR oppose cependant [a] et [a] en syllabe finale ouverte, comme dans rat [ra] vs ras [ra], alors que la variété québécoise généralise la voyelle postérieure [a] dans cette position, sauf dans certains mots grammaticaux (ex. $\grave{a}, l a, c ̧ a$ proclitique, qui s'opposent à $a$, là, ça tonique) et les formes rédupliquées (ex. papa, caca). Enfin, devant [r] final, la distinction [a] [a] est neutralisée en faveur de [a] dans le PR, mais en faveur de [a] en français québécois. Des mots comme barre et tard sont donc transcrits [bar] et [tar] dans le PR, mais prononcés [bar] et [tar] au Québec ${ }^{16}$.

L'usage québécois diverge également des transcriptions du PR sur la distribution des voyelles moyennes en syllabe non finale. Ces voyelles sont notamment soumises à trois facteurs:

- l'harmonie vocalique, selon laquelle le timbre des voyelles non finales tend vers celui de la voyelle finale de mot;

- la loi de position, qui favorise les voyelles mi-fermées [e ø o] en syllabe ouverte et les voyelles mi-ouvertes $[\varepsilon \propto \rho]$ en syllabe fermée;

- l'analogie, qui favorise le maintien, dans les formes morphologiquement dérivées, de la voyelle dans la forme de base.

Pour une forme comme blesser dérivée de blesse [bles], l'harmonie vocalique et la loi de position favorisent [blese] et l'analogie [blese]. Alors que le PR tend à respecter l'harmonie vocalique et la loi de position, c'est l'analogie qui s'impose dans l'usage québécois.

Enfin, la prononciation des séquences voyelle fermée + voyelle varie grandement dans le monde francophone (Durand et Lyche 1999). Le PR applique systématiquement la synérèse, c'est-à-dire la prononciation de ces séquences en une syllabe par la semi-vocalisation de la voyelle fermée: muet

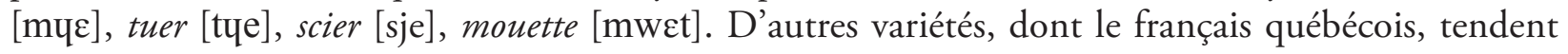
davantage vers la diérèse, soit la prononciation des mêmes séquences en deux syllabes: muet [mye], tuer [tye], scier [sie], mouette [mu\&t]. De multiples facteurs interviennent ici - identité de la voyelle fermée, structure morphologique du mot, nature des consonnes environnantes -, mais la diérèse est quasi-systématique au Québec avec [y] (muet), à l'exception de < ui > [ui] (puis) (Côté à paraitre; Kelly 2014).

Dans quelle mesure les notes de prononciation reflètent-elles l'usage québécois sur ces points? La réponse est simple: aucunement, le Multi se conformant presque complètement aux tendances observées dans le PR. Pour ce qui est de la distribution de [a] et [a] en syllabe finale, le Multi précise que le mot poêle «se prononce comme poil [pwal]), ce qui est tout à fait contraire à l'usage québécois. Tous les mots contenant en syllabe finale la suite orthographique < ar > et qui bénéficient d'une note de prononciation sont transcrits [ar] plutôt que [ar] (ex. jaguar, poignard, dossard, etc.). Enfin, en finale de mot, le Multi reprend largement la répartition entre [a] et [a] qu'on trouve dans le PR, [a] étant nettement majoritaire. Sur les 120 mots concernés, seuls 5 s'écartent de la transcription du PR

16. La généralisation de $[\mathrm{a}]$ devant $[\mathrm{r}]$ final au Québec connaît toutefois quelques exceptions lexicales (prépare, gare, guitare et quelques autres), présentement en voie de disparition (Côté 2012). 
en optant pour [a] plutôt que [a] (fatras, glas, raz, cervelas, compas). Le Multi s'éloigne donc en fait de l'usage québécois en systématisant la tendance au [a] final ${ }^{17}$.

Les voyelles moyennes en syllabe non finale sont un des traits sur lesquels le Multidictionnaire insiste le plus, comme on l'a vu dans la section 3. Dans les formes où s'opposent l'harmonie vocalique, la loi de position et l'analogie, le Multi s'aligne encore sur le PR, y compris dans les cas où l'usage québécois diffère nettement. Les notes pour asséner, desseller, greffé et les autres dérivés de greffe et quereller recommandent donc [e] en syllabe pénultième, à l'encontre de l'usage québécois, qui favorise le maintien $\mathrm{du}[\varepsilon]$ issu des formes de base assène, selle, greffe et querelle. Pour bêtise, blêmir, blêmissement, grêler et guêpier, l'usage québécois impose [3], que l'on trouve aussi dans bête, blême, grêle et guêpe. Le Multi ne peut rendre compte de ces prononciations puisqu'il ne considère pas la voyelle [3]. Mais, au lieu de se tourner vers $[\varepsilon]$, il recommande explicitement [e].

Qu'en est-il, enfin, de la diérèse et de la synérèse dans les séquences voyelle fermée + voyelle? Tout comme le PR, le Multi applique assez systématiquement la synérèse, comme dans diesel [djezcl], sciemment [sjamã] et arguer [argчe], que les Québécois prononcent volontiers en trois syllabes plutôt que deux (en ordre croissant de probabilité dans cette séquence de trois mots). Cet aspect de la prononciation ne fait cependant l'objet d'aucun commentaire explicite.

Les emprunts à l'anglais ne sont pas toujours intégrés de la même façon dans les variétés de français parlées des deux côtés de l'Atlantique. Puisque les emprunts sont particulièrement bien représentés dans les "difficultés de prononciation", il y a lieu de se demander si le Multidictionnaire les traite différemment du vocabulaire natif. Nous avons identifié huit tendances régulières dans l'adaptation des mots anglais qui distinguent le français québécois de la norme européenne, telle que reflétée dans le PR:

- adaptation de la voyelle anglaise $[\Lambda]$ en [œ $\tilde{\mathfrak{e}} \sim \mathrm{u}]$ en Europe vs [९] au Québec: lunch [1œnt $\int$ lõ $\left.\int\right]$ vs [lonf], brushing [brœein] vs [brofın] ${ }^{18}$;

- adaptation du [o:] anglais en [o] (parfois [a]) en Europe vs [a] au Québec: football [futbol] vs [futbal], handball [ãdbal] vs [endbal];

- adaptation du suffixe anglais -er en [er] en Europe vs [œr] (voire [əo]) au Québec: scooter [skuter] vs [skutœr] ; [œr] s'observe aussi en Europe;

- adaptation de l'anglais [er] en [ع] (parfois [a]) en Europe vs [e] au Québec: break [brek] vs [brek], babyfoot [babifut] vs [bebifut];

- adaptation du [i] final de mot anglais en [i] en Europe vs [e] au Québec: party [parti] vs [parte];

- adaptation de la terminaison -on en [on] en Europe vs [œn] (ou [Yn]) au Québec: badminton [badminton] vs [badmintœn];

- adaptation de l'anglais VN (voyelle orale + consonne nasale) en Ṽ en Europe vs VN au Québec: bang [bãg] vs [bạ]; VN s'observe aussi en Europe;

- adaptation de l'anglais [ou] en [o] en Europe vs [o] au Québec: dope [dəp] vs [dop].

17. Le Multi fait rimer les mots entrelacs, gars, haras et tétra, tous en [a] dans le Multi et le PR, avec la, méga, rat et intra. Cette deuxième série de mots, dont le Multi ne note pas la prononciation, est pourtant transcrite [a] dans le PR, ce qui montre bien l'incohérence du traitement de ces voyelles finales.

18. Le PR indique une nasale vélaire [n] dans les emprunts en -ing, par opposition à une palatale [n] dans le lexique français (ex. ligne). À notre connaissance, cette opposition n'est pas faite au Québec. On y observe cependant une variation générale entre [n] et [y] en fin de mot (Côté 2012). Nous ne notons que [y] dans les emprunts, comme le Multi et le PR. 
Les emprunts à l'anglais n'adoptent cependant pas nécessairement au Québec tous les traits cidessus, dépendant de leur histoire lexicale. Un mot comme rugby, par exemple, est normalement prononcé [rygbi] plutôt que [rogbe].

Nous avons identifié 68 emprunts à l'anglais qui font l'objet d'une note de prononciation dans le Multi et pour lesquels on peut considérer que la prononciation dominante au Québec suit ces tendances; 81 traits de prononciation illustrent les huit tendances ci-dessus. Les transcriptions du PR suivent généralement les tendances européennes; elles peuvent également accepter les deux prononciations, voire, dans de rares cas, ne recommander que la prononciation qui correspond aussi à la tendance dominante au Québec. Nous avons classé ces 81 traits de prononciation selon les transcriptions du PR et du Multi: tendance européenne seulement, tendance "québécoise» seulement, les deux (tableau 3). Chaque combinaison est donnée avec les traits (soulignés) qui l'illustrent.

Tableau 3: Transcriptions de 68 emprunts à l'anglais dans le Multi et le Petit Robert

\begin{tabular}{|c|c|c|c|c|}
\hline & \multicolumn{3}{|c|}{ Transcriptions dans le Petit Robert } \\
\hline & & $\begin{array}{l}\text { Européenne } \\
\text { seulement }\end{array}$ & Les deux & Québécoise seulement \\
\hline \multirow{3}{*}{ 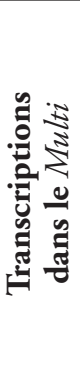 } & $\begin{array}{l}\text { Européenne } \\
\text { seulement }\end{array}$ & $\begin{array}{c}56 \\
\text { chutney, cross-country, } \\
\text { volleyball, boxer, badminton, } \\
\text { brandy }\end{array}$ & $\begin{array}{c}4 \\
\text { challenge, challenger, jamboree, } \\
\text { manager }\end{array}$ & $\begin{array}{c}3 \\
\text { lady, challenger, squatter }\end{array}$ \\
\hline & Les deux & $\begin{array}{c}6 \\
\text { gangster, ketch } \underline{u} p, \text { lobby, plum- } \\
\text { pudding, poney, pupb }\end{array}$ & $\begin{array}{c}3 \\
\text { blazer, bulldozer, scooter }\end{array}$ & - \\
\hline & $\begin{array}{l}\text { Québécoise } \\
\text { seulement }\end{array}$ & $\begin{array}{c}4 \\
\text { babyfoot, bacon, } \operatorname{bog}(g) \underline{\text { ie }}\end{array}$ & bulldozer, gentleman & $\begin{array}{c}3 \\
\text { leader, leadership, retriever }\end{array}$ \\
\hline
\end{tabular}

Ce tableau montre que le Multi ne reflète généralement pas l'usage québécois. On y observe une adaptation vers la prononciation québécoise dans trois cas de figure: présence des deux variantes - alors que le PR ne donne que l'européenne - et présence de la seule variante québécoise - alors que le PR indique soit l'européenne soit les deux variantes. En tout, ce sont douze cas sur 81 (15\%) qui sont concernés; ils apparaissent en gris clair dans le tableau. Dans sept cas ( $9 \%$, en grisé foncé dans le tableau), le Multi s'éloigne au contraire de l'usage québécois en ne retenant que la variante européenne, alors que la variante en usage au Québec apparaît dans le PR. Dans une bonne majorité des cas (69 \%, 56 sur 81), les transcriptions du Multi correspondent à celles du PR et ignorent la variante québécoise dominante.

\subsection{La légitimité des prononciations québécoises}

Il n'est pas possible de savoir, à la lumière de ce qui précède, si l'auteure du Multi ignore tout simplement certains traits de prononciations propres à l'usage québécois ou si, au contraire, le traitement insuffisant de la prononciation témoigne d'un parti pris négatif à leur endroit. Toujours est-il que, en passant sous silence plusieurs traits courants au Québec, l'ouvrage contribue à les discréditer, même lorsqu'ils font partie du registre soigné.

Le peu de prestige accordé aux prononciations québécoises est encore plus apparent lorsqu’on regarde les prononciations explicitement condamnées. Ces condamnations rappellent directement l'orientation normative de l'ouvrage. Pour la plupart d'entre elles $(85 / 113$ cas), il s'agit de traits 
proprement québécois appartenant soit au registre soigné, soit au registre familier (ou, dans quelques rares cas, à la langue populaire). Le Multidictionnaire condamne par exemple la prononciation [ $\varepsilon]$ (au lieu de [e]) dans la première syllabe d'effacé et de ressusciter, la prononciation avec [z] (au lieu de [s]) des mots dysentérie et israélien ou encore la prononciation [œ] (au lieu de [ø]) dans peuplier. Il s'agit pourtant de prononciations tout à fait courantes au Québec, y compris dans le registre soigné. Parmi les prononciations relevant du registre familier ou de la langue populaire, le Multidictionnaire condamne dehors [deor], haïssable [agisabl], pyjama [pidzama] ou encore shampooing [ ãpu].

\section{Conclusion}

Malgré sa prétention de décrire le bon usage québécois, le Multidictionnaire ne tient compte que très imparfaitement des phénomènes de prononciation bien attestés dans le registre soigné au Québec. Les Québécois seraient pourtant en droit de retrouver ces traits dans un dictionnaire dont l'ambition est de décrire leur usage. Dans la grande majorité des cas où la prononciation québécoise s'écarte de celle qui est indiquée dans le Petit Robert, le Multidictionnaire ne reflète pas ces différences. Nous estimons que dans l'ensemble des notes sur la prononciation, au moins une transcription sur trois bénéficierait d'une révision, ce qui est considérable.

De toute évidence, la prononciation québécoise souffre d'un manque de légitimité dans le Multidictionnaire qui, dans les faits, véhicule un modèle normatif dont les repères se trouvent essentiellement en France. Les quelques ouvertures à l'usage québécois, loin de contribuer à en faire valoir la légitimité, n’y changent rien. En faisant cohabiter des variantes québécoises et européennes, le Multidictionnaire véhicule plutôt une image incomplète de la variété québécoise. La position normative qui en résulte est incohérente et ne peut que laisser l'utilisateur déconcerté. Sur ce point, nos observations rejoignent les constats faits par d'autres à propos de la position normative du Multi en matière de lexique.

\section{Références bibliographiques}

Bergeron, G. 1998. «Le Multi-dictionnaire: de jeunesse et d'ambition». Québec français 111: 83-84. Consulté le 16 mars 2018 de http://id.erudit.org/iderudit/56291ac.

Bigot, D. et R. A. Papen. 2013. "Sur la "norme” du français oral au Québec (et au Canada en général) ». Langage et société 146: 115-132. doi : http://dx.doi.org/10.3917/ls.146.0115.

Bouchard, P., B. Harmegnies, M.-L. Moreau, A. Prikhodkine et S. Pascal. 2004. «La norme dans la francophonie périphérique: externe ou interne? Une étude expérimentale au Québec, en Belgique et en Suisse». Dans La variation dans la langue standard, sous la direction de P. Bouchard. Québec: Office québécois de la langue française: 51-71.

Boughton, Z. 2015. "Social class, cluster simplfication and following context: Sociolinguistic variation in wordfinal post-obstruent liquid deletion in French". Journal of French Language Studies 25: 1-21. doi: http:// dx.doi.org/10.1017/S0959269513000446.

Cajolet-Laganière, H. et P. Martel. 2013. Usito. Sherbrooke: Éditions Delismes. [http://www.usito.com]

Corbeil, J.-C. 2004. «Le Multi: un dictionnaire pragmatique». Québec français 134: 26-27. Consulté le 16 mars 2018 de http://id.erudit.org/iderudit/55570ac.

Corbeil, J.-C. 2015. «Préface». Dans Multidictionnaire, conçu et rédigé par M.-É. de Villers. Montréal: Québec Amérique: xi-xii. 
Cormier, M. 2008. "Genèse et évolution du Multidictionnaire de la langue française». Dans Les dictionnaires de la langue française au Québec: de la Nouvelle-France à aujourd'hui, sous la direction de M. C. Cormier et J.-C. Boulanger. Montréal: Presses de l'Université de Montréal : 291-307.

Côté, M.-H. 2009. "Contraintes segmentales et variation dans la perte et la stabilisation du schwa en syllabe initiale». Dans Le français d'un continent à l'autre, sous la direction de L. Baronian et F. Martineau. Québec: Presses de l'Université Laval: 93-121.

Côté, M.-H. 2012. "Laurentian French (Québec) : Extra vowels, missing schwas and surprising liaison consonants". Dans Phonological Variation in French: Illustrations from Three Continents, sous la direction de R. Gess, C. Lyche et T. Meisenburg. Amsterdam: John Benjamins: 235-274.

Côté, M.-H. À paraître. "Glissantes et diphtongues en français laurentien ». Cahiers de l'Institut de Linguistique et des Sciences du Langage, Université de Lausanne.

Cox, T. 1998. "Vers une norme pour un cours de phonétique française au Canada». La Revue canadienne des langues vivantes 54 (2): 172-197. doi : http://dx.doi.org/10.3138/cmlr.54.2.172.

De Villers, M.-É. 1988. Multidictionnaire des difficultés de la langue française, $1^{\text {re }}$ édition. Montréal: Québec Amérique.

De Villers, M.-É. 2015. Multidictionnaire de la langue française, 6e édition. Montréal: Québec Amérique.

Durand, J. et C. Lyche. 1999. "Regard sur les glissantes en français: français standard, français du Midi». Cahiers de grammaire 24: 39-65. Consulté le 16 mars 2018 de http://w3.erss.univ-tlse2.fr/textes/ publications/CDG/24/CG24-4-Durand.pdf.

Evans, B. 2002. "Attitudes of Montreal students towards varieties of French". Dans Handbook of Perceptual Dialectology, vol. 2, sous la direction de D. Long et D. R. Preston. Amsterdam/Philadelphie: John Benjamins: 71-93.

Gendron, J.-D. 2014. La modernisation de l'accent québécois. De l'accent traditionnel au nouvel accent: 1841 1960. Esquisse historique. Contribution à l'histoire de la prononciation du français au Québec. Québec: Presses de l'Université Laval.

Jousselin, J.-P. 1989. "Le Multidictionnaire des difficultés de la langue française». Québec français 73: 62-63. Consulté le 16 mars 2018 de http://id.erudit.org/iderudit/45277ac.

Kelly, S. 2014. Variable Glide Formation in Hexagonal French. Thèse de doctorat, University of Western Ontario. Le Petit Robert de la langue française 2016. Paris: Dictionnaires Le Robert.

Milne, P. 2014. The Variable Pronunciations of Word-Final Consonant Clusters in a Force Aligned Corpus of Spoken French. Thèse de doctorat, Université d'Ottawa.

Moreau, M.-L., P. Bouchard, S. Demattin, F. Gadet, E. Guerin, B. Harmegnies, K. Huet, F. Laroussi, A. Prikhodkine, P. Singy, N. Thiam et H. Tyne. 2007. Les accents dans la francophonie: une enquête internationale. Fernelmont/Bruxelles: Éditions E.M.E./Service de la langue française.

Ostiguy, L. et C. Tousignant. 2008. Les prononciations du français québécois: normes et usages, 2e édition. Montréal: Guérin universitaire.

Paradis, C., J. Dolbec et V. Arnaud. 2008. Phono: les principales caractéristiques du français parlé au Québec. Québec/Chicoutimi: Université Laval/Université du Québec à Chicoutimi. Consulté le 16 mars 2018 de http://phono.uqac.ca.

Poirier, C. 1998. Dictionnaire historique du français québécois. Sainte-Foy: Presses de l'Université Laval.

Poirier, C. 2004. "Le Multi: un dictionnaire ambigu». Québec français 132: 26-27. Consulté le 16 mars 2018 de http://id.erudit.org/iderudit/55632ac.

Poirier, C. et S. Canac-Marquis. 2003. «La norme du "Multi" dans le "Petit Larousse”?». Le Soleil 8 septembre: A3.

Séguin, A. 2013. La pratique définitoire du Multidictionnaire de la langue française: le traitement des noms de plantes alimentaires. Mémoire de maîtrise, Université de Sherbrooke. 
Thibault, A. 2013. «Les transcriptions phonétiques/phonologiques des diatopismes du français dans la lexicographie». Dans La variation du français dans les espaces créolophones et francophones, tome 2 , sous la direction de G. Ledegen. Paris: L'Harmattan : 151-176.

Thibault, A. et L. Thibault. 1993. "Compte rendu du Dictionnaire du français plus et du Dictionnaire québécois d'aujourd'hui». Revue de linguistique romane 57: 577-581. 